\title{
PARAMETRIC STUDY OF THROUGHPUT PERFORMANCE IN SBS/RS BASED ON SIMULATION
}

\author{
Lerher, T.; Borovinsek, M.; Ficko, M. \& Palcic, I. \\ University of Maribor, Faculty of Mechanical Engineering, Smetanova 17, 2000 Maribor, Slovenia \\ E-Mail: tone.lerher@um.si,matej.borovinsek@um.si,mirko.ficko@um.si,iztok.palcic@um.si
}

\begin{abstract}
This paper presents an analysis of cycle times and throughput performances of Shuttle-Based Storage and Retrieval Systems (SBS/RSs), a relatively new technology that is becoming an important part of automated warehouses. A parametric simulation model was developed for the purposes of this research. The model enables calculation of Single Command (SC) and Dual Command (DC) cycle times and throughput performances of SBS/RS. The model was tested on a case study, which is based on a real type of SBS/RS. Experiments were designed to evaluate the following factors, such as number of bays $n_{x}$ and minimum warehouse volume $Q$. Our research uses Design of Experiment (DOE) analysis and helps to achieve the proper dimension of the SBS/RS, which gives us the best possible performance of the SBS/RS. The simulation based on the presented model delivers the best SBS/RS designs.

(Received in May 2016, accepted in October 2016. This paper was with the authors 2 months for 1 revision.)
\end{abstract}

Key Words: Logistics, Warehouses, Shuttle-Based Storage and Retrieval Systems, Simulation, Design of Experiments, Performance Analysis

\section{INTRODUCTION}

The Internet based technologies have enabled the possibility to have better control of virtually all processes and to be able to customize mass produced goods. Customers accepted these new possibilities very well which, in turn, caused the reduction of production series`sizes and increased the number of different products. The need for management of a large number of variants, in smaller quantities, has arisen. All activities which support manufacturing processes have to follow this trend, including the activities of warehousing, logistics and transport. If, in the first phase, the computerization of warehouses was the sufficient answer to new demands, in the next phase, the changed supplier and customer needs demand construction of new types of warehouses. The need for storage and retrieval equipment has also changed because of the change of technologies. One of the answers to these challenges are SBS/RS, which are rather new and, therefore, need to be researched with various approaches.

An SBS/RS consists of shuttle carriers, elevators with a lifting table and storage racks. The most important difference between an SBS/RS and an Automated Storage and Retrieval System (AS/RS) is the way storage and retrieval devices move [1]. In an AS/RS cranes that move simultaneously in horizontal and vertical directions handle the unit loads. In an SBS/RS the unit loads are handled by autonomous vehicles (shuttle carriers) moving horizontally, and by an elevator (with lifting table) moving vertically [2]. Autonomous vehicles enable simultaneous operating of many shuttle carriers at the same time, therefore, theoretically, doubling the throughput performance. One shuttle carrier can operate at extreme conditions at each tier. Of course, in this way, we get a bottleneck at the lifting table, since it has to serve for vertical movement for all the used shuttles. SBS/RSs have more design variables, which need to be taken into account at design. In addition to the common AS/RS variables such as rack width and height, we have variables such as the number or shuttles, number of elevator lifting tables, the elevator position, and the buffer position. 
During the design process, the designers have to search for near optimal solutions from the large number of possible storage configurations for the one which offers the best performance. This problem is presented and researched in this article; the final goal was to find the best (near optimal) design of SBS/RS. Since it is impossible to get an analytical solution due to the large number of variables, we recognised the simulation as the best option to search and validate the SBS/RS design.

This paper is organised in the following way. A literature review on SBS/RSs is presented in Section 2. The model for designing an SBS/RS is presented in Section 3. A simulation based parametric case study is presented in Section 4. Finally, conclusions are drawn in Section 5.

\section{LITERATURE REVIEW}

In recent years, many companies have invested in intensive automated warehousing systems to keep their logistics activities more flexible and efficient [3-5]. Automated warehousing systems have been used for the storage and retrieval of products in both distribution and production environments since the 1960s. The implementation of such systems has increased considerably during the last decades given their several advantages over non-automated systems [6-8]. The SBS/RS is a subset of an Autonomous Vehicle Storage and Retrieval System (AVS/RS) [1]. SBS/RSs are relatively new technology and are becoming an important part of automated warehouses, used widely in many fields of industry [9-11].

An AVS/RS technology has been introduced into scientific literature by Malmborg [12]. In his performance analysis study with a reference to a tier-to-tier configuration, he proposed a queuing model to estimate the cycle time performance considering the number of columns, tiers, vehicles, and lifts. On the other hand, ones of the first who analysed SBS/RS were Carlo and Vis [13]. They studied a type of SBS/RS with two non-passing lifting systems mounted along the rack. They focused on a scheduling problem where two functions were introduced to evaluate solution performances. SBS/RS problems were approached with different methods. For example, Marchet et al. [14] presented an open queuing network approach to design an analytical model to estimate some performance measures (waiting and cycle time of transactions) to study SBS/RS and compared their experimental results with simulation results. Epp et al. [15] presented a method for the calculation of the performance measures of a tier-captive single-aisle AVS/RS with general discrete inter-arrival and service times, modelled as a discrete-time open queuing network.

Simulation is another method used to evaluate the performance analysis of specific SBS/RS configurations, since it can yield a more realistic approach compared to the analytical model [16]. According to Ning et al. [1] the reason is the fact that a deterministic (analytical) approach usually requires simplifications of the model that are not required in a (stochastic) simulation model.

Ekren and Heragu [17] have studied a simulation-based performance evaluation of AVS/RS. Their study deals with near optimum rack configuration design under pre-defined scenarios of number of vehicles and lifts in the system by using simulation-based regression analysis. They have developed 30 simulation models for six design scenarios by using ARENA software. Ekren and Heragu [18] also used simulation to determine near optimum values for a number of autonomous vehicles and lifts; seven design scenarios, and 133 different simulation experiments were performed in this study. Later, Marchet et al. [19] presented main design trade-offs for SBS/RS by using simulation. They completed their study for several warehouse design scenarios for tier-captive shuttle vehicles and proposed a design framework that can be used to optimise cost structure and system performance for the predefined SBS/RS designs. Ekren [20] proposed a graph-based solution for performance 
evaluation of an AVS/RS under various design concepts, that included the number of bays, aisles and tiers for the rack design and arrival rate of $S / R$ transactions to an aisle of the warehouse. The performance of the system was evaluated in terms of the key performance measures from the system: Utilisations of lifts and average cycle time of S/R transactions, where simulation was utilised for the modelling purpose. Zou et al. [21] proposed a parallel processing policy for tier-captive AVS/RS, under which the lift and the vehicles can serve a transaction simultaneously. To investigate the performance of the parallel processing policy, they formulated a fork-join queueing network and built simulation models to validate the analytical model. The results showed that the analytical model could estimate the performance of tier-captive AVS/RS accurately under the parallel processing policy. Ning et al. [1] developed a model for simulating the rack configuration of a multi-elevator tier-captive SBS/RS. They used a simulation case study for a multi-elevator tier-captive SBS/RS containing 81 different storage rack alternatives that were simulated under 15 different retrieval rates. Based on the simulation results, the optimal design solution was identified, considering two objectives: Maximise the throughput and minimise the transaction cycle time.

Lerher et al. have performed many research studies on SBS/RS. For example, Lerher et al. [22] presented an analytical travel time model for the computation of travel (cycle) times for SBS/RS. The proposed models provide mean travel time estimations for SC and DC cycles, where simulation models tested the performance of the developed models. Lerher [23] also presented an analytical travel time model for the computation of cycle times for double-deep SBS/RS that demonstrated good performances for evaluating double-deep SBS/RS. These studies were upgraded by Borovinšek et al. [24], where the authors proposed a multi objective optimization solution procedure for design of an SBS/RS by using the NSGA II genetic algorithm for facilitating the solution. The procedure considers three objective functions in the design concept: Minimization of the average cycle time of transactions (average throughput time), amount of energy (electricity) consumption and total investment cost. Finally, Lerher et al. [25] used a simulation tool to evaluate the performance of an SBS/RS by utilising the detailed modelling ability of it. The results showed that SBS/RS are effective in reducing the mean cycle time and showed large improvement by increasing throughput capacity.

Different from the existing studies, the previous SBS/RS model, e.g. [25], will be extended by implementing a Design of Experiment (DOE) analysis for the selected SBS/RS. We found only one research based on the use of DOE that deals with AVS/RS, which aims to identify factors affecting their performance [26, 27]. Our research uses DOE analysis and helps to achieve the proper dimension of the SBS/RS along with the kinematic properties of the elevators' lifting table and shuttle carriers, which gives us the best possible performance of the SBS/RS.

\section{MODEL FOR DESIGNING AN SBS/RS}

A typical installation of an SBS/RS consists of a storage rack for totes, with an elevator with lifting table and shuttle carriers, which are operating in each tier of the SBS/RS (Fig. 1). The unit loads (totes) are handled by autonomous vehicles (shuttle carriers) moving horizontally and by an elevator (with lifting table) moving vertically. Therefore, a number of storage and retrieval commands can be performed simultaneously in an SBS/RS and better performance may be achieved [1]. Loads are stored and removed from the shelves at high speed, since the shuttle's load handling attachments are designed for short pick-up and set-down times [20]. The elevator through the lifting table moves totes up and down to the prescribed tier in the storage rack. To be able to process more work two independent lifting tables may be attached to the elevator, one of which is on the right side and the other one is on the left side of the 
mast. In this case, the performance of the elevator can be doubled. Theoretically, an elevator can move lifting tables with a velocity up to $5 \mathrm{~m} / \mathrm{s}$. Elevators are usually a bottleneck in this system so they affect the throughput rate of the system and usually determine the performance of the whole system [28].

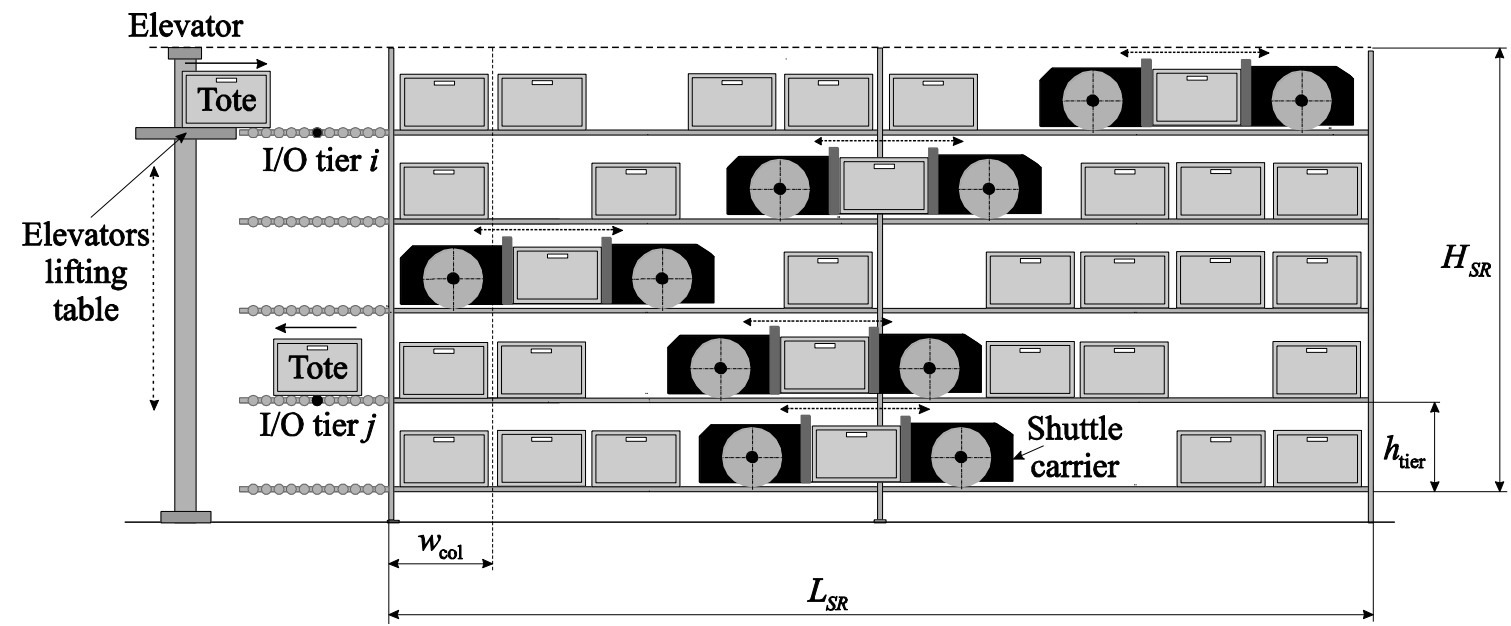

Figure 1: Shuttle-Based Storage and Retrieval Systems (tier-captive shuttle carriers).

The following notations and symbols are found in Fig. 1:

- $H_{\mathrm{SR}}$ - storage rack height,

- $L_{\mathrm{SR}}$ - storage rack length,

- $h_{\text {tier }}-$ tier height,

- I/O - input/output location,

- $w_{\text {col }}$ - column width.

A shuttle carrier is a small autonomous vehicle with four wheels that transports totes from the buffer position to the storage locations on the storage rack (Fig. 1). A shuttle carrier includes a telescopic attachment for totes manipulation. The maximal weight of a tote is $50 \mathrm{~kg}$ per shuttle carrier. Theoretically, a shuttle carrier maximum travel velocity is $4 \mathrm{~m} / \mathrm{s}$. Usually there is a single shuttle carrier in each level of the storage rack. This assumption can be released if we use a special shuttle elevator at the back of the storage rack, for moving shuttle carriers up and down to the prescribed level of the storage rack. A buffer position is placed at each level of the storage rack and is used for buffering totes from the elevator and shuttle carriers. The storage rack is composed of columns and tiers. The capacity of the storage rack is calculated by multiplying columns in the horizontal and tiers in the vertical directions [28].

The performance of an SBS/RS is determined by the total amount of their movement in a specific time period (throughput capacity of the system). The number of required storage locations depends on the designed warehouse capacity. The warehouse management system monitors the status of material handling components in the system (elevators' lifting tables, shuttle carriers, buffer positions, etc.). The work to be carried out is planned based on the order list and movement requirements. The elevator consists of a vertical mast or a pair of masts supporting the lifting table that are moving vertically. The elevator's lifting table is feeding the buffer positions, which are set at the beginning of each tier of the SBS/RS. In each tier of the SR there is a shuttle carrier that can store and retrieve totes [23].

The installation of an SBS/RS with one lifting table works on the basis of SC or DC cycles. The application of the DC cycle is more efficient, since the throughput capacities are comparing to the SC cycle, higher. In this case, totes arrive in the system on the right side of the SBS/RS and are departing the system on the left side of the SBS/RS. 


\subsection{Simulation model of an SBS/RS}

The simulation model of the SBS/RS is based on the algorithm presented in Fig. 2. The simulation model consists of three main modules and several decisions and calculation routines. The first module, "SBS/RS geometry", includes the selection of the basic transport unit load (tote) for items' handling. Based on the selected transport unit load, the storage cell is determined, which forms the basis for setting up the storage rack. According to the required warehouse volume $(Q)$, dimensions of the storage rack $\left(L_{\mathrm{SR}}\right.$ and $\left.H_{\mathrm{SR}}\right)$ for the single aisle SBS/RS are determined. In the second module, "Velocity scenarios", the velocity profile of the elevator's lifting table ( $v_{y}$ and $\left.a_{y}\right)$ and the velocity profile of the shuttle carriers $\left(v_{x}\right.$ and $\left.a_{x}\right)$ are defined. Pick-up and set-down times $\left(t_{\mathrm{P} / \mathrm{S}}\right)$ of the elevator's lifting table and the shuttle carrier with the relationship between the number of SC and DC cycles are defined in the module "Other input data". The maximum number of runs $\left(i_{\max }\right)$ needs to be defined before the simulation.

The simulation loop begins with the decision module $(i=1)$ where the condition "probSC" < "scTasks" is defined. When the variable "scTasks" equals 1, then, according to the Boolean data type, only SC cycles will be executed. On the other hand, when variable "scTasks" equals 0, then, according to the Boolean data type, only DC cycles will be executed. Next, according to the programme code written in the VBA, SC cycle times $T(\mathrm{SC})$ and DC cycle times $T$ (DC) are calculated (Fig. 2). If the condition $i+1<i_{\max }$ holds true, then the same loop in executed once again. When $i+1$ equals $i_{\max }$, then the average SC and DC cycle times $(\overline{T(\mathrm{SC})}, \overline{T(\mathrm{DC})})$ along with throughput performances $(\overline{\lambda(\mathrm{SC})}, \overline{\lambda(\mathrm{DC})})$ are calculated, respectively.

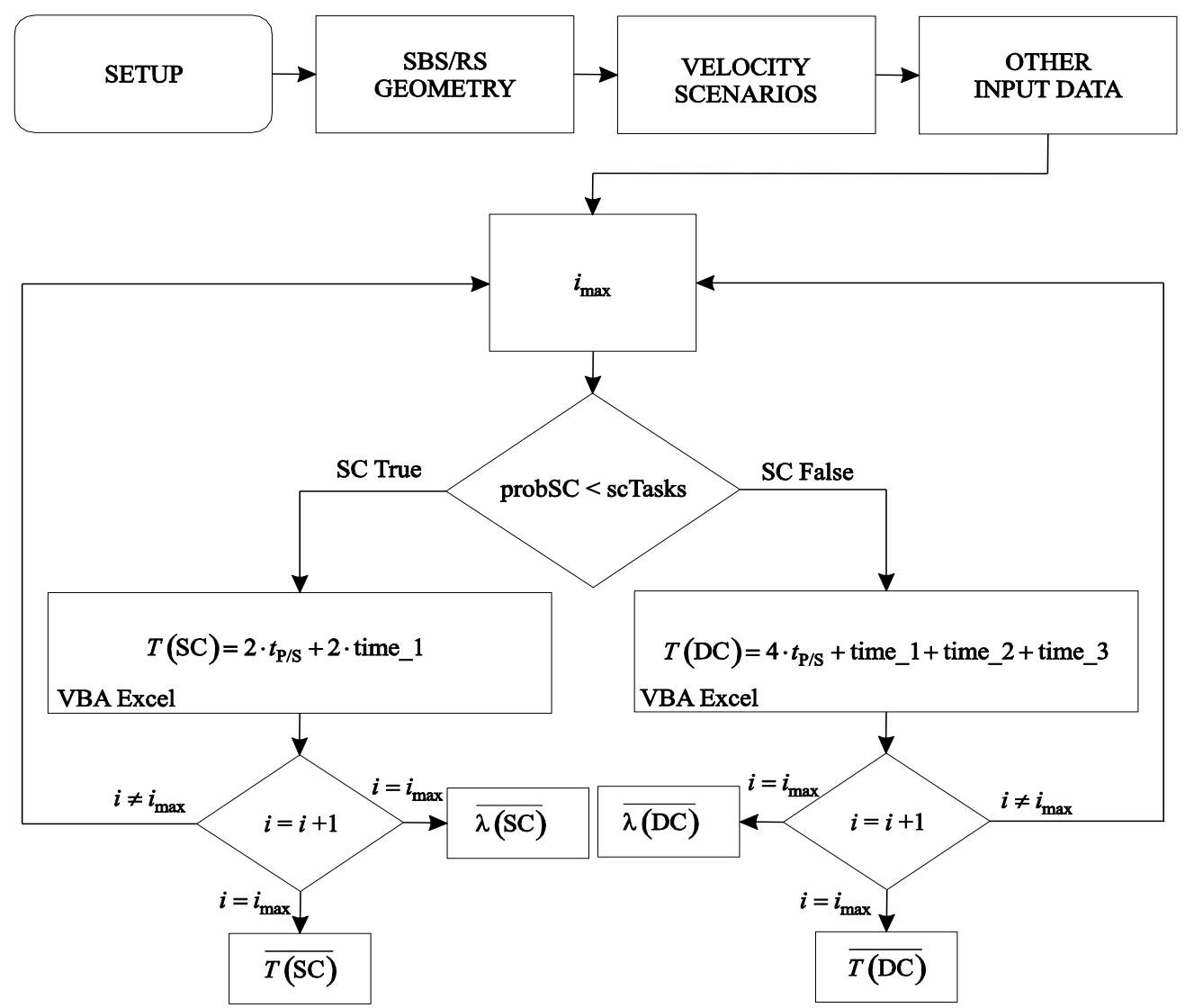

Figure 2: Algorithm of the simulation model of an SBS/RS.

The detailed algorithm for calculating SC and DC cycle times and throughput performances of the shuttle carrier is presented in Fig. 3. 


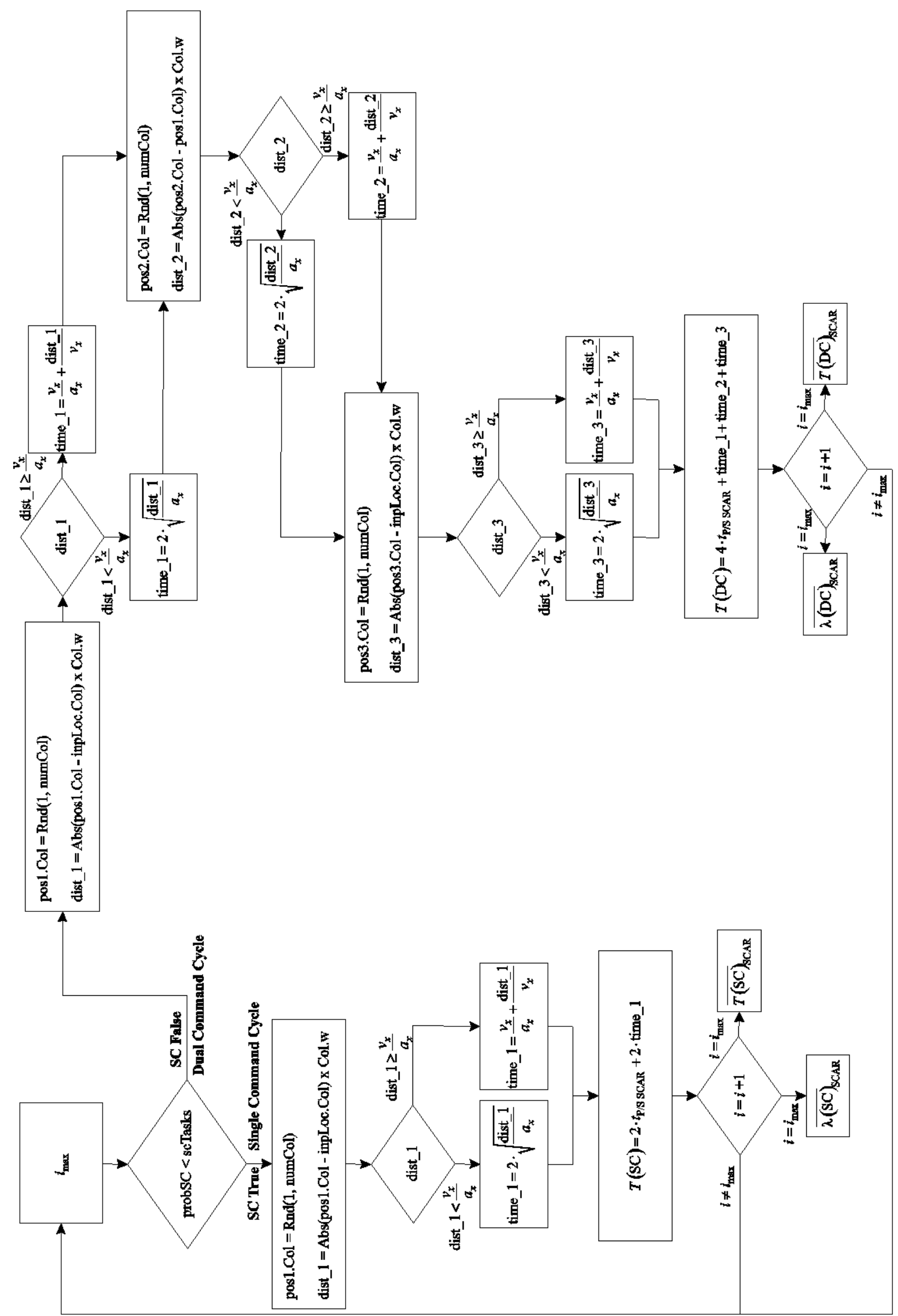

Figure 3: Algorithm for calculating SC and DC cycle times and throughput performances of the shuttle carrier. 
The algorithm in Fig. 3 works in the following way. If variable "scTasks" equals 1, then, according to the Boolean data type, only SC cycles will be executed. According to the maximum number of columns of the SBS/RS (numCol), the current column position "pos1.Col" is selected randomly. Next, the variable "dist_1" is calculated according to the position of the I/O location and the column width ("Col.w"). When the variable "dist_1" $<v 2 / a$, then the time "time_1" equals the expression (1):

$$
\text { time_1 }=2 \sqrt{\frac{\text { dist_1 }}{a}}
$$

When the variable "dist_1" $\geq v^{2} / a$, then the time "time_1" equals the expression (2):

$$
\text { time } 1=\frac{v}{a}+\frac{\text { dist_}_{-} 1}{v}
$$

Next, SC cycle time $T(\mathrm{SC})_{\mathrm{SCAR}}$ is calculated by using the expression (3):

$$
T(\mathrm{SC})_{\mathrm{SCAR}}=2 \cdot t_{\mathrm{P} / \mathrm{S}}+2 \cdot \text { time_}_{-} 1
$$

When $i+1$ equals $i_{\max }$, then the average $\mathrm{SC}$ cycle time $\overline{T(\mathrm{SC})}$ SCAR and the average throughput performance $\overline{\lambda(\mathrm{SC})}$ SCAR equal the expression (4):

$$
\begin{aligned}
& \overline{T(\mathrm{SC})}_{\mathrm{SCAR}}=\frac{\sum_{i=1}^{i_{\max }} T(\mathrm{SC})_{\mathrm{SCAR}}}{i_{\max }}[\mathrm{sec} .] \\
& \overline{\lambda(\mathrm{SC})}_{\mathrm{SCAR}}=\frac{3600}{\overline{T(\mathrm{SC})})_{\mathrm{SCAR}}}[\text { totes/hour] }
\end{aligned}
$$

The average DC cycle time $\overline{T(D C)}_{\text {SCAR }}$ and the average throughput performance $\overline{\lambda(D C)}_{\text {SCAR }}$ of the shuttle carrier are calculated in a similar way.

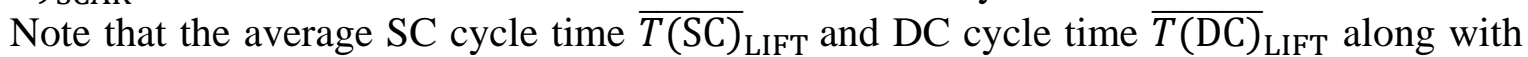
the average throughput performances $\overline{\lambda(\mathrm{SC})}_{\mathrm{LIFT}}$ and $\overline{\lambda(\mathrm{DC})}_{\mathrm{LIFT}}$ of the elevator's lifting table are calculated by using the same equations with different variables (tier height, number of tiers, $v, a$, etc.).

\subsection{Design of experiment}

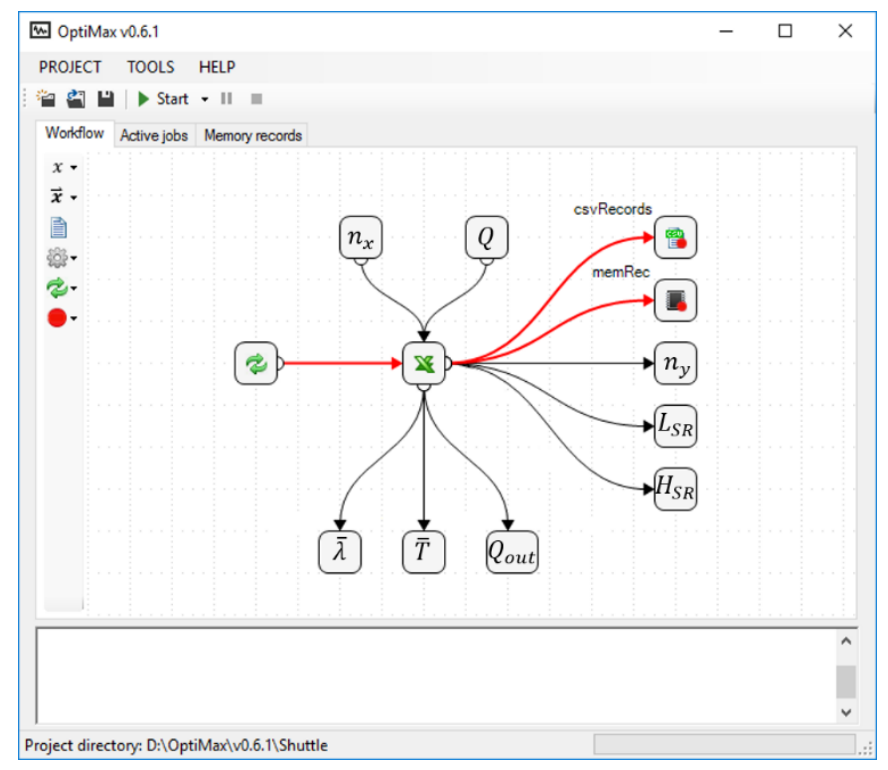

Figure 4: Design of experiment with OptiMax. 
Design of Experiment, or DOE, is a design tool that analyses the relation between independent (input) and dependent (output) variables in order to identify the significant factors affecting the output. To identify the significant factors affecting the performance of an SBS/RS, five performance measures (Fig. 4) were considered, as follows: Average single command $\overline{T(\mathrm{SC})}$ and average dual command $\overline{T(\mathrm{DC})}$ cycle times of the shuttle carrier and the elevator's lifting table, average throughput performances $\overline{\lambda(\mathrm{SC})}$ and $\overline{\lambda(\mathrm{DC})}$ of the shuttle carrier and the elevator's lifting table and the warehouse volume $Q_{\text {out }}$. We considered two factors in the DOE (Fig. 4) that are likely to have an effect on these performance measures: Number of bays $n_{x}$ and minimum warehouse volume $Q$. The DOE procedure was done by using OptiMax software.

\section{SIMULATION BASED PARAMETRIC STUDY}

In our simulation model, the following geometrical parameters have been used: Tote's length $l_{\text {tote }}=0.6 \mathrm{~m}$, tote's width $w_{\text {tote }}=0.4 \mathrm{~m}$, tote's height $h_{\text {tote }}=0.24 \mathrm{~m}$, column's length $($ depth) $l_{\text {col }}=0.6 \mathrm{~m}$, column's width $w_{\text {col }}=0.5 \mathrm{~m}$ and tier's height $h_{\text {tier }}=0,42 \mathrm{~m}$. Warehouse volume was set to a minimum of 2,000 totes. Minimum length $L_{\text {MIN }}$ of the storage rack was set to 20 $\mathrm{m}$, while the maximum length $L_{\mathrm{MAX}}$ of the storage rack was set to $100 \mathrm{~m}$. Minimum height $H_{\text {MIN }}$ of the storage rack was set to $2.1 \mathrm{~m}$ and the maximum height $H_{\mathrm{MAX}}$ of the storage rack was set to $10.5 \mathrm{~m}$. The velocity scenario of the elevator's lifting table and the shuttle carrier is presented in Table I.

Table I: The velocity scenario of the elevator's lifting table and the shuttle carrier.

\begin{tabular}{|c|c|c|c|c|c|c|}
\hline \multirow{2}{*}{$\begin{array}{c}\text { Velocity } \\
\text { scenario }\end{array}$} & \multicolumn{3}{|c|}{$\begin{array}{c}\text { Shuttle carrier travelling in the horizontal } \\
\text { direction }\end{array}$} & \multicolumn{3}{|c|}{$\begin{array}{c}\text { Elevator's lifting table movement in the } \\
\text { vertical direction }\end{array}$} \\
\cline { 2 - 7 } & $v_{x}$ & $a_{x}^{+}$ & $a_{x}^{-}$ & $v_{y}$ & $a_{y}^{+}$ & $a_{y}^{-}$ \\
\hline 1 & 2.0 & 2.0 & 2.0 & 4.0 & 5.0 & 5.0 \\
\hline
\end{tabular}

Pick-up and set-down times of the elevator's lifting table and the shuttle carrier were set to $t_{\mathrm{P} / \mathrm{S} \mathrm{SCAR}}=3.0 \mathrm{sec}$ and $t_{\mathrm{P} / \mathrm{S} \mathrm{LIFT}}=1.5 \mathrm{sec}$, respectively. In order to receive the best (average) result, the simulation based parametric study was conducted with 30,000 runs and ten individual replication runs for each combination type of SBS/RS.

\subsection{Analysis and evaluation of results}

Tables II and III give details of the designed full factorial DOE experiment undertaken to determine the relative importance of the factors and their interactions.

Table II: The best experimental combinations for the SC cycle.

\begin{tabular}{|c|c|c|c|c|c|}
\hline \multirow{2}{*}{ Output } & \multirow{2}{*}{$\begin{array}{c}\text { Best } \\
\text { experiment }\end{array}$} & $n_{x}$ & $L_{\mathrm{SR}}$ & $n_{y}$ & $H_{\mathrm{SR}}$ \\
\cline { 3 - 6 } & $18.23 \mathrm{sec}$ & 40 & 20 & 25 & 10.50 \\
\hline$\overline{T(\mathrm{SC})}_{\text {SCAR }}$ & $4.43 \mathrm{sec}$ & 200 & 100 & 5 & 2.10 \\
\hline$\overline{T(\mathrm{SC})}_{\mathrm{LIFT}}$ & & 40 & 20 & 25 & 10.50 \\
\hline$\overline{\lambda(\mathrm{SC})}_{\text {SCAR }}$ & ${ }^{*} 4937$ totes/h & 200 & 100 & 5 & 2.10 \\
\hline$\overline{\lambda(\mathrm{SC})}_{\mathrm{LIFT}}$ & 813 totes/h & 198 & 99 & 6 & 2.52 \\
\hline$Q$ & 2376 totes & 198 & & & \\
\hline
\end{tabular}

Note: ${ }^{*} \overline{\lambda(\mathrm{SC})}_{\mathrm{SCAR}}=\left(3600 / \overline{T(\mathrm{SC})}_{\mathrm{SCAR}}\right) \cdot n_{y}$ 
Table III: The best experimental combinations for the DC cycle.

\begin{tabular}{|c|c|c|c|c|c|}
\hline \multirow{2}{*}{ Output } & Best & \multicolumn{5}{|c|}{ Factor levels } \\
\cline { 3 - 6 } & experiment & $n_{x}$ & $L_{\mathrm{SR}}$ & $n_{y}$ & $H_{\mathrm{SR}}$ \\
\hline$\overline{T(\mathrm{DC})}_{\text {SCAR }}$ & $28.65 \mathrm{sec}$ & 40 & 20 & 25 & 10.50 \\
\hline$\overline{T(\mathrm{DC})}_{\text {LIFT }}$ & $8.22 \mathrm{sec}$ & 200 & 100 & 5 & 2.10 \\
\hline$\overline{\lambda(\mathrm{DC})}_{\text {SCAR }}$ & ${ }^{* *} 6283$ totes/h & 40 & 20 & 25 & 10.50 \\
\hline$\overline{\lambda(\mathrm{DC})}_{\text {LIFT }}$ & 876 totes $/ \mathrm{h}$ & 200 & 100 & 5 & 2.10 \\
\hline$Q$ & 2376 totes & 198 & 99 & 6 & 2.52 \\
\hline
\end{tabular}

Note: ${ }^{* *} \overline{\lambda(\mathrm{DC})}_{\mathrm{SCAR}}=\left(3600 / \overline{T(\mathrm{DC})}_{\mathrm{SCAR}}\right) \cdot 2 \cdot n_{y}$

According to Tables II and III, the shuttle carrier performs well in the case of relatively short storage racks. The best throughput performance $\left(\overline{\lambda(\mathrm{SC}}_{\mathrm{SCAR}}=4,937\right.$ totes/hour and $\overline{\lambda(\mathrm{DC})}_{\mathrm{SCAR}}=6,283$ totes/hour) lies within a small number of bays $\left(n_{x}=40\right)$ and large number of tiers $\left(n_{y}=25\right)$. In this case, we get a relatively high $\left(n_{y}=25\right)$ and short $\left(n_{x}=40\right)$ storage rack, which is not the best solution from the elevator's performance perspective. On the contrary, the elevator's lifting table performs well in the case of relatively small storage racks. The best throughput performance $\left(\overline{\lambda(\mathrm{SC}}_{\mathrm{LIFT}}=813\right.$ totes/hour and $\overline{\lambda(\mathrm{DC})}_{\mathrm{LIFT}}=876$ totes/hour) lies within a small number of tiers $\left(n_{y}=5\right)$ and large number of bays $\left(n_{x}=200\right)$. In this case, we get a relatively small $\left(n_{y}=5\right)$ and long $\left(n_{x}=200\right)$ storage rack, which is not the best solution from the shuttle carrier`s performance perspective. The best performance of the SBS/RS will be by crossing curves (Fig. 5) of the elevator's lifting table and the shuttle carriers, where the elevator's lifting table and the shuttle carriers perform with the highest utilization $\left(\eta_{\text {LIFT }}\right.$ and $\left.\eta_{\text {SCAR }} \sim 1.0\right)$.

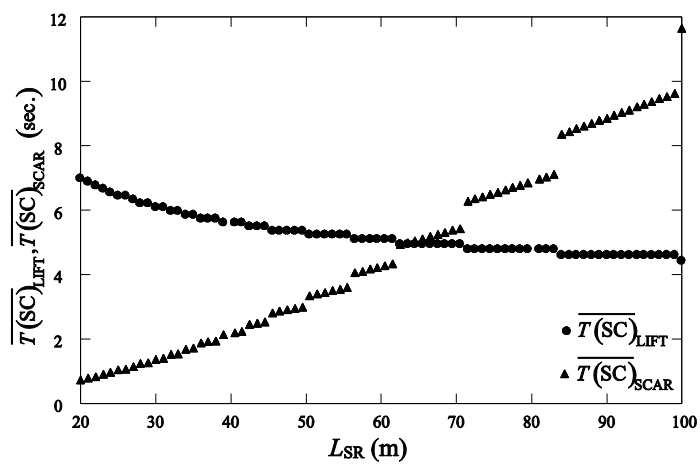

a) $\overline{T(\mathrm{SC})}_{\mathrm{LIFT}}, \overline{T(\mathrm{SC})}_{\mathrm{SCAR}}$

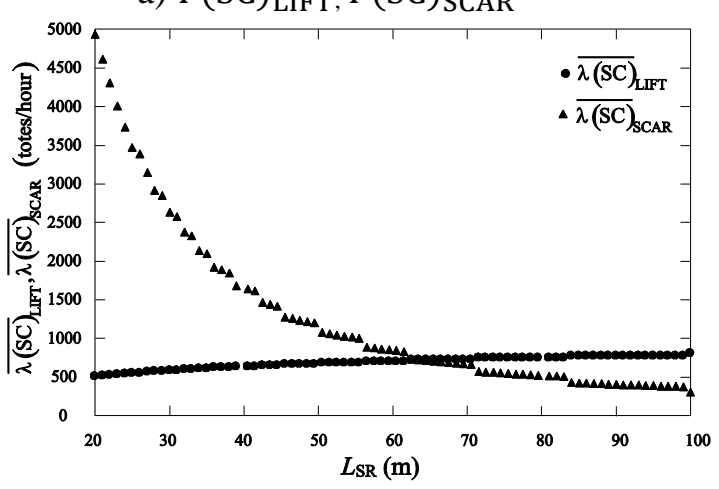

c) $\overline{\lambda(\mathrm{SC})}_{\mathrm{LIFT}}, \overline{\lambda(\mathrm{SC})}_{\mathrm{SCAR}}$

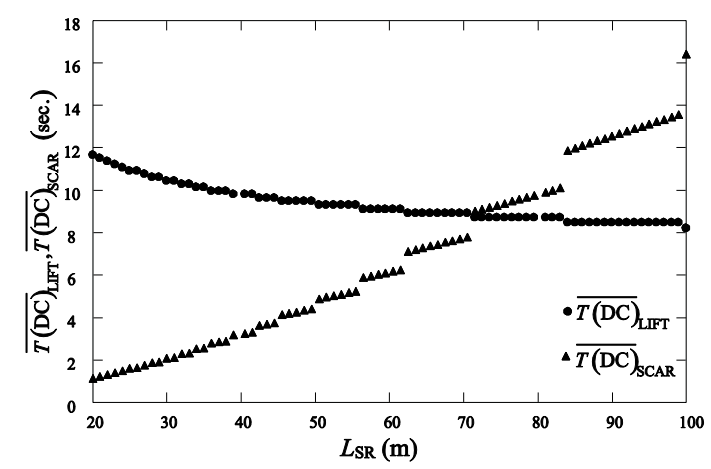

b) ${\overline{T(D C})_{\mathrm{LIFT}}, \overline{T(\mathrm{DC})}}_{\mathrm{SCAR}}$

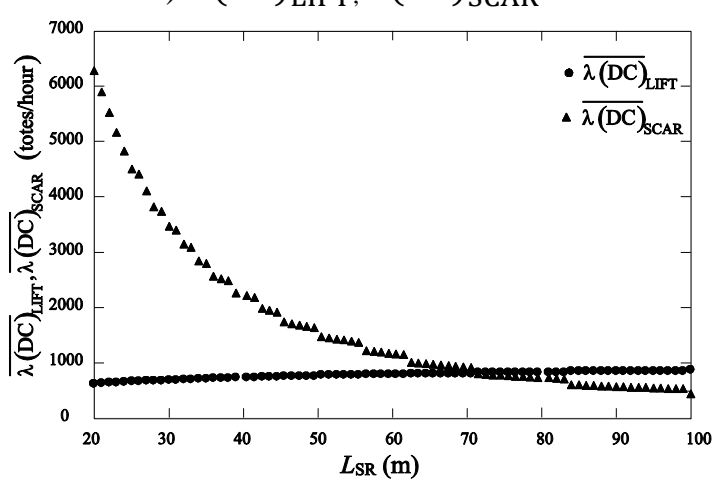

d) $\overline{\lambda(\mathrm{DC})}_{\mathrm{LIFT}}, \overline{\lambda(\mathrm{DC})}{ }_{\mathrm{SCAR}}$

Figure 5: Cycle times and throughput performances`comparison of the elevator's lifting table and shuttle carriers. 
Note: Because of the configuration of the SBS/RS, the average SC and DC cycle times of the shuttle carrier are divided by the number of tiers. This means that in each tier there is a single shuttle carrier (tier-captive system) that is executing tasks in the horizontal direction (in parallel), meanwhile the elevator's lifting table is executing tasks in the vertical direction (sequential).

Based on the results in Figs. 5 a, 5 b, 5 c and 5 d, the best solution for the SC cycle lies within the storage rack with the following dimensions: $L_{\mathrm{SR}}=62.5 \mathrm{~m}\left(n_{x}=125\right)$ and $H_{\mathrm{SR}}=3.36 \mathrm{~m}\left(n_{y}=8\right)$, where throughput performances of the elevator's lifting table $\overline{\lambda(\mathrm{SC})}_{\mathrm{LIFT}}=726$ totes/hour and shuttle carriers $\overline{\lambda(\mathrm{SC})}_{\mathrm{SCAR}}=730$ totes/hour are relatively close.

The best solution for the DC cycle lies within the storage rack with the following dimensions: $L_{\mathrm{SR}}=71.5 \mathrm{~m}\left(n_{x}=143\right)$ and $H_{\mathrm{SR}}=2.94 \mathrm{~m}\left(n_{y}=7\right)$, where throughput performances of the elevator's lifting table $\overline{\lambda(\mathrm{DC})}_{\mathrm{LIFT}}=827$ totes/hour and shuttle carriers $\overline{\lambda(\mathrm{DC})}_{\mathrm{SCAR}}=800$ totes/hour are relatively close.

\section{CONCLUSION}

In this paper, a parametric study based on simulation was proposed in order to identify the best throughput performance of an SBS/RS. First, a simulation model of an SBS/RS, which was developed in MS Excel by using the visual basic for application programme language, was described and discussed in detail. Next, the design of the experiment was presented and discussed using a special software tool called OptiMax.

Factors that could affect the performance measures from the SBS/RS were defined to be the number of bays $n_{x}$ and minimum warehouse volume $Q$. The performance measures were considered as follows: Average single command $\overline{T(\mathrm{SC})}$ and average dual command $\overline{T(\mathrm{DC})}$ cycle times of the shuttle carrier and the elevator's lifting table, average throughput performances $\overline{\lambda(\mathrm{SC})}$ and $\overline{\lambda(\mathrm{DC})}$ of the shuttle carrier and the elevator's lifting table and the warehouse volume $Q_{\text {out }}$. Results of the parametric study are summarised in Tables II, III and in Fig. 5.

It can be concluded that warehouse designers aiming to minimise the average cycle time of the elevator's lifting table and of the shuttle carriers should consider designing the SBS/RS with long storage racks $\left(\gg n_{x}\right)$ and relatively small numbers of tiers $n_{y}$. The best solution of the SBS/RS for a DC cycle lies within the storage rack with the following dimensions: $n_{x}=143\left(L_{\mathrm{SR}}=71.5 \mathrm{~m}\right)$ and $n_{y}=7\left(H_{\mathrm{SR}}=2.94 \mathrm{~m}\right)$, where throughput performances of the elevator's lifting table $\overline{\lambda(\mathrm{DC})}_{\mathrm{LIFT}}=827$ totes/hour and shuttle carriers $\overline{\lambda(\mathrm{DC})}_{\mathrm{SCAR}}=800$ totes/hour are relatively close. The latter means that the elevator's lifting table and the shuttle carriers will operate with maximum utilization $(\eta \approx 1.0)$, which is good from the investment point of view.

This study can be extended in many directions. For example: $(i)$ Different velocity and acceleration / deceleration scenarios for the elevator's lifting table and the shuttle carrier, (ii) Different configurations of the SBS/RS, (iii) Application of the environmental aspects like energy consumption and $\mathrm{CO}_{2}$ emissions may also be considered in the analysis. It would also be interesting to investigate the class-based policy (ABC zoning) on the throughput performance of the SBS/RS.

\section{ACKNOWLEDGEMENT}

The authors acknowledge the financial support from the Slovenian Research Agency (research core funding No. P2-0157 and No. P2-0190). 


\section{REFERENCES}

[1] Ning, Z.; Lei, L.; Saipeng, Z.; Lodewijks, G. (2016). An efficient simulation model for rack design in multi-elevator shuttle-based storage and retrieval systems, Simulation Modelling Practice and Theory, Vol. 67, 100-116, doi:10.1016/j.simpat.2016.03.007

[2] Fukunari, M.; Malmborg, C. J. (2009). A network queuing approach for evaluation of performance measures in autonomous vehicle storage and retrieval systems, European Journal of Operations Research, Vol. 193, No. 1, 152-167, doi:10.1016/j.ejor.2007.10.049

[3] Azzi, A.; Battini, D.; Faccio, M.; Persona, A.; Sgarbossa, F. (2011). Innovative travel time model for dual-shuttle automated storage/retrieval systems, Computers \& Industrial Engineering, Vol. 61, No. 3, 600-607, doi:10.1016/j.cie.2011.04.015

[4] Vujica-Herzog, N.; Tonchia, S.; Polajnar, A. (2009). Linkages between manufacturing strategy, benchmarking, performance measurement and business process reengineering, Computers \& Industrial Engineering, Vol. 57, No. 3, 963-975, doi:10.1016/j.cie.2009.03.015

[5] Karabegović, I.; Karabegović, E.; Mahmić, M.; Husak, E. (2015). The application of service robots for logistics in manufacturing processes, Advances in Production Engineering \& Management, Vol. 10, No. 4, 185-194, doi:10.14743/apem2015.4.201

[6] Wauters, T.; Villa, F.; Christiaens, J.; Alvarez-Valdes, R.; Vanden Berghe, G. (2016). A decomposition approach to dual shuttle automated storage and retrieval systems, Computers \& Industrial Engineering, Vol. 101, 325-337, doi:10.1016/j.cie.2016.09.013

[7] Vujičić, A.; Zrnić, N.; Jerman, B. (2013). Ports sustainability: A life cycle assessment of zero emission cargo handling equipment, Strojniški vestnik - Journal of Mechanical Engineering, Vol. 59, No. 9, 547-555, doi:10.5545/sv-jme.2012.933

[8] Marinović, I.; Sprečić, D.; Jerman, B. (2012). A slewing crane payload dynamics, Tehnički vjesnik - Technical Gazette, Vol. 19, No. 4, 907-916

[9] Koren, R.; Prester, J.; Buchmeister, B.; Palčič, I. (2016). Do organisational innovations have impact on launching new products on the market?, Strojniški vestnik - Journal of Mechanical Engineering, Vol. 62, No. 6, 389-397, doi:10.5545/sv-jme.2016.3470

[10] Stanković, R.; Šafran, M.; Božić, D. (2016). Guidelines for improving logistic performances as drivers of the logistic industry development, Tehnički vjesnik - Technical Gazette, Vol. 23, No. 5, 1497-1503, doi:10.17559/TV-20141016184435

[11] Chen, Y. X. (2016). Integrated optimization model for production planning and scheduling with logistics constraints, International Journal of Simulation Modelling, Vol. 15, No. 4, 711-720, doi:10.2507/IJSIMM15(4)CO16

[12] Malmborg, C. J. (2002). Conceptualizing tools for autonomous vehicle storage and retrieval systems, International Journal of Production Research, Vol. 40, No. 8, 1807-1822, doi: $\underline{10.1080 / 00207540110118668}$

[13] Carlo, H. J.; Vis, I. F. A. (2012). Sequencing dynamic storage systems with multiple lifts and shuttles, International Journal of Production Economics, Vol. 140, No. 2, 844-853, doi:10.1016/j.ijpe.2012.06.035

[14] Marchet, G.; Melacini, M.; Perotti, S.; Tappia, E. (2012). Analytical model to estimate performances of autonomous vehicle storage and retrieval systems for product totes, International Journal of Production Research, Vol. 50, No. 24, 7134-7148, doi: $10.1080 / 00207543.2011 .639815$

[15] Epp, M.; Wiedemann, S.; Furmans, K. (2017). A discrete-time queueing network approach to performance evaluation of autonomous vehicle storage and retrieval systems, International Journal of Production Research, Vol. 55, No. 4, doi:10.1080/00207543.2016.1208371

[16] Seebacher, G.; Winkler, H.; Oberegger, B. (2015). In-plant logistics efficiency valuation using discrete event simulation, International Journal of Simulation Modelling, Vol. 14, No. 1, 60-70, doi:10.2507/IJSIMM14(1)6.289

[17] Ekren, B. Y.; Heragu, S. S. (2010). Simulation-based regression analysis for the rack configuration of an autonomous vehicle storage and retrieval system, International Journal of Production Research, Vol. 48, No. 21, 6257-6274, doi:10.1080/00207540903321665 
[18] Ekren, B. Y.; Heragu, S. S. (2011). Simulation based performance analysis of an autonomous vehicle storage and retrieval system, Simulation Modelling Practice and Theory, Vol. 19, No. 7, 1640-1650, doi:10.1016/j.simpat.2011.02.008

[19] Marchet, G.; Melacini, M.; Perotti, S.; Tappia, E. (2013). Development of a framework for the design of autonomous vehicle storage and retrieval systems, International Journal of Production Research, Vol. 51, No. 14, 4365-4387, doi:10.1080/00207543.2013.778430

[20] Ekren, B. Y. Graph-based solution for performance evaluation of shuttle-based storage and retrieval system, International Journal of Production Research, Article in Press, 11 pages, doi: $10.1080 / 00207543.2016 .1203076$

[21] Zou, B.; Xu, X.; Gong, Y. Y.; De Koster, R. (2016). Modelling parallel movement of lifts and vehicles in tier-captive vehicle-based warehousing systems, European Journal of Operational Research, Vol. 254, No. 1, 51-67, doi:10.1016/j.ejor.2016.03.039

[22] Lerher, T.; Ekren, B. Y.; Dukic, G.; Rosi, B. (2015). Travel time model for shuttle-based storage and retrieval systems, The International Journal of Advanced Manufacturing Technology, Vol. 78, No. 9-12, 1705-1725, doi:10.1007/s00170-014-6726-2

[23] Lerher, T. (2016). Travel time model for double-deep shuttle-based storage and retrieval systems, International Journal of Production Research, Vol. 54, No. 9, 2519-2540, doi:10.1080/ 00207543.2015 .1061717

[24] Borovinšek, M.; Ekren, B. Y.; Burinskienè, A.; Lerher, T. (2016). Multi-objective optimisation model of shuttle-based storage and retrieval system, Transport, Article in Press, doi: $10.3846 / 16484142.2016 .1186732$

[25] Lerher, T.; Ekren, Y. B.; Sari, Z.; Rosi, B. (2015). Simulation analysis of shuttle based storage and retrieval systems, International Journal of Simulation Modelling, Vol. 14, No. 1, 48-59, doi:10.2507/IJSIMM14(1)5.281

[26] Ekren, B. Y.; Heragu, S. S.; Krishnamurthy, A.; Malmborg, C. J. (2010). Simulation based experimental design to identify factors affecting performance of AVS/RS, Computers \& Industrial Engineering, Vol. 58, No. 1, 175-185, doi:10.1016/j.cie.2009.10.004

[27] Tang, M.; Gong, D.; Liu, S.; Zhang, H. (2016). Applying multi-phase particle swarm optimization to solve bulk cargo port scheduling problem, Advances in Production Engineering \& Management, Vol. 11, No. 4, 299-310, doi:10.14743/apem2016.4.228

[28] Lerher, T.; Ekren, B. Y.; Sari, Z.; Rosi, B. (2016). Method for evaluating the throughput performance of shuttle based storage and retrieval systems, Tehnicki vjesnik - Technical Gazette, Vol. 23, No. 3, 715-723, doi:10.17559/TV-20141022121007 